\title{
Effects of Somatostatin Combined with Pantoprazole on Serum C-Reactive Protein and Intercellular Adhesion Molecule-1 in Severe Acute Pancreatitis
}

\author{
Jun Liu, Guoliang Wang, Yan Liu, Lei Huang, Xiangang Xu and Jun Wang
}

\begin{abstract}
The objective of this study was to investigate effects of somatostatin combined with pantoprazole on serum C-reactive protein (CRP) and intercellular adhesion molecule-1 (ICAM-1) in severe acute pancreatitis (SAP) patients. It was an experimental study carried out from February 2016 to April 2018. Eighty-two patients were randomly divided into group A and group B with 41 in each group. Pantoprazole was used in group A and somatostatin combined with pantoprazole was used in group $B$. Results showed that time of abdominal pain relief, intestinal function recovery and ventilator weaning in group $B$ were shorter than those in group $A$ (all $p<0.001$ ). After treatment, levels of CRP and ICAM-1 in group B were lower than those in group $A$ (both $p<0.001$ ). Compared with pantoprazole alone, somatostatin combined with pantoprazole has a better therapeutic effect on SAP, and its mechanism may be related to reduction of serum CRP and ICAM- 1 .
\end{abstract}

Key Words: Somatostatin, Pantoprazole, Severe acute pancreatitis, C-reactive protein, Intercellular adhesion molecule-1.

Severe acute pancreatitis (SAP) is a critical disease caused by diffuse hemorrhage of the pancreas and combined with necrosis of surrounding tissues. ${ }^{1}$ Conventional symptomatic treatments such as pain relief, gastrointestinal pressure reduction, correction of water and electrolyte and acid-base disorder play an important role in controlling the secretion of gastric juice and pancreatic juice in patients with severe acute pancreatitis, but the therapeutic effect is not ideal. Somatostatin is a kind of peptide hormone, which has an effect on the inhibition of the secretion of growth hormone and gastrointestinal hormone. Studies have shown that early use of somatostatin can alleviate inflammation and improve immune function in SAP patients, but the impact on mortality and morbidity of patients needs further study. ${ }^{2}$ Pantoprazole is a new proton pump inhibitor, which can reduce the secretion of gastric acid and pepsin and effectively improve the gastrointestinal $\mathrm{PH}$ value of patients, thus blocking the secretion of physiological protease, reducing the secretion of pancreas body, and exerting a high acidsuppressing effect. Pantoprazole can reduce inflammatory cell infiltration and necrosis of acinar cells in SAP patients, which has been shown in a study. ${ }^{3}$ Serum levels of C-reactive protein (CRP) and intercellular adhesion molecule-1 (ICAM-1) are important experimental indi-

Department of Hepatobiliary Surgery, Guizhou Province

People's Hospital, Guiyang City, Guizhou Province, 550002,

China

Correspondence: Dr. Jun Liu, Department of Hepatobiliary

Surgery, Guizhou Province People's Hospital, Guiyang City,

Guizhou Province, 550002, China

E-mail:egqlk8@163.com

Received: December 21, 2018; Revised: February 21, 2019;

Accepted: February 21, 2019 cators for judging the severity of pancreatitis and detecting pancreatic necrosis. The objective of this study was to investigate the effects of somatostatin combined with pantoprazole on serum C-reactive protein and intercellular adhesion molecule-1 in patients with severe acute pancreatitis.

This experimental study was carried out at the Department of Hepatobiliary Surgery, Guizhou Province People's Hospital, China, from February 2016 to April 2018. A total of 82 SAP patients were enrolled in this study. The inclusion criteria were that patients diagnosed as SAP, over 18 years old, and the time from onset to admission was less than 48 hours. Patients with severe heart, lung, liver and kidney diseases, Diabetes, hematological diseases and severe hyperlipidemia and patients who took immunosuppressive agents and hormones for a long time were excluded. The patients were randomly divided into group A and group B with 41 cases in each group. Both groups were treated with fasting, maintaining acid-base balance and electrolyte balance, gastrointestinal decompression, cefuroxime and levofloxacin to control infection and inhibiting pancreatic secretion. Nutritional support and blood volume supplementation were used. Group A was treated with pantoprazole, i.e. $40 \mathrm{mg}$ pantoprazole was added into $5 \% 100 \mathrm{~mL}$ of glucose injection and intravenously dripped twice a day. Group B was treated with somatostatin combined with pantoprazole, i.e. on the basis of group A, somatostatin was added. For the first time, $250 \mu \mathrm{g}$ somatostatin was injected intra-venously, and then $3000 \mu \mathrm{g}$ somatostatin $+500 \mathrm{~mL} 0.9 \%$ sodium chloride injection was given by micro-intravenous pump at a speed of $250 \mu \mathrm{g}$ per hour, twice a day. The course of treatment in both groups was 7 days. 
Table I: Comparison of related indicators between the two groups.

\begin{tabular}{l|c|c|c}
\hline Parameter & $\begin{array}{c}\text { Group A } \\
(\mathrm{n}=41)\end{array}$ & $\begin{array}{c}\text { Group B } \\
(\mathrm{n}=41)\end{array}$ & p-value \\
\hline Time of abdominal pain relief $(\mathrm{d})$ & $3.93 \pm 0.57$ & $2.17 \pm 0.64$ & $<0.001$ \\
Time of intestinal function recovery $(\mathrm{d})$ & $4.72 \pm 0.66$ & $3.05 \pm 0.51$ & $<0.001$ \\
Time of ventilator weaning $(\mathrm{d})$ & $5.58 \pm 1.34$ & $3.63 \pm 0.92$ & $<0.001$ \\
Before treatment serum CRP $(\mathrm{mg} / \mathrm{L})$ & $145.24 \pm 7.85$ & $146.03 \pm 8.19$ & 0.657 \\
After treatment serum CRP $(\mathrm{mg} / \mathrm{L})$ & $128.15 \pm 5.26$ & $109.58 \pm 3.77$ & $<0.001$ \\
Before treatment serum ICAM-1 $(\mathrm{ng} / \mathrm{mL})$ & $12.67 \pm 3.42$ & $12.84 \pm 3.95$ & 0.835 \\
After treatment serum ICAM-1 $(\mathrm{ng} / \mathrm{mL})$ & $9.14 \pm 1.81$ & $7.06 \pm 1.13$ & $<0.001$ \\
\hline
\end{tabular}

The time of abdominal pain relief, intestinal function recovery, and ventilator weaning were observed. Blood samples were taken from elbow vein before and after the treatment. Serum CRP and ICAM-1 levels were measured after serum separation. Serum CRP was detected by immunoturbidimetry. Serum ICAM-1 was detected by ELISA.

Data was analysed using SPSS version 25. Numerical data were expressed as mean and standard deviation and examined by independent sample t-test. Qualitative data were expressed as frequencies and percentages. $\mathrm{P}$-value of less than or equal to 0.05 was considered statistically significant.

Among the 82 patients, 48 (58.54\%) were males and 34 $(41.46 \%)$ were females. The age was ranged from 31 to 64 with an average age of $55.76 \pm 2.93$ years. The time of visiting a doctor was from 1.2 to 5.9 hours with an average time of $3.74 \pm 1.25$ hours.

The time of abdominal pain relief, intestinal function recovery and ventilator weaning in group $B$ were shorter than those in group A (all $p<0.001$, Table I). Before treatment, there was no significant difference in the levels of serum CRP and ICAM-1 between the two groups ( $p=0.657,0.835)$, while the levels of serum CRP and ICAM-1 in group B were lower than those in group A (both $p<0.001$, Table I) after treatment.

Some studies have found that somatostatin is more effective than pantoprazole in inhibiting gastric acid secretion in patients with peptic ulcer bleeding. ${ }^{4}$ As the action mechanisms of somatostatin and pantoprazole are different, the combination of the two may play a better therapeutic effect. Therefore, this study investigated the therapeutic effect of somatostatin combined with pantoprazole sodium in the treatment of SAP. This study showed that the time of abdominal pain relief, intestinal function recovery and ventilator weaning in group B were better than those in group A. It is suggested that the combination of somatostatin and pantoprazole has a better therapeutic effect on SAP.

Halonen et al. have shown that excessive release of inflammatory mediators and cell factors play an important role in the development of SAP. ${ }^{5}$ CRP is a marker of body injury or acute or chronic inflammation. ICAM-1 can mediate adhesion of cell-to-cell or cell-toextracellular matrix and play an important role in many biological processes such as inflammation. Studies have shown that serum ICAM-1 is an early diagnostic and predictive indicator of SAP. 6 The results showed that somatostatin combined with pantoprazole could effectively reduce serum CRP and ICAM-1 levels in SAP patients, which might be a mechanism that somatostatin combined with pantoprazole could effectively improve SAP symptoms.

\section{REFERENCES}

1. Thomson JE, Nweke EE, Brand M, Nel M, Candy GP, Fonteh PN. Transient expression of interleukin-21 in the second hit of acute pancreatitis may potentiate immune paresis in severe acute pancreatitis. Pancreas 2019; 48:107-12.

2. Tang WF, Wan MH, Zhu L, Chen GY, Xia Q, Huang X. Immunomodulatory effect of somatostatin combined with traditional Chinese medicine on severe acute pancreatitis at early stage: A randomized control trial. J Chin Integr Med 2005; 3:103-7.

3. Hackert T, Tudor S, Felix K, Dovshanskiy D, Hartwig W, Simon WA, et al. Effects of pantoprazole in experimental acute pancreatitis. Life Sci 2010; 87:551-7.

4. Avgerinos A, Sgouros S, Viazis N, Vlachogiannakos J, Papaxoinis K, Bergele C, et al. Somatostatin inhibits gastric acid secretion more effectively than pantoprazole in patients with peptic ulcer bleeding: A prospective, randomized, placebo-controlled trial. Scand J Gastroenterol 2005; 40: 515-22.

5. Halonen KI, Pettilä V, Leppäniemi AK, Kemppainen EA, Puolakkainen PA, Haapiainen RK. Multiple organ dysfunction associated with severe acute pancreatitis. Crit Care Med 2002; 30:1274-9.

6. Zhu HH, Jiang LL. Serum inter-cellular adhesion molecule 1 is an early marker of diagnosis and prediction of severe acute pancreatitis. World J Gastroenterol 2012; 18:2554-60. 